\title{
Estimating Insurance Premiums using Credibility Theory and Quantiles
}

\author{
Li Yang*
}

\begin{abstract}
This thesis is based on the paper 'Quantile credibility models' by Georgios Pitselis, which was published in 2013. It introduces credibility theory and shows how quantiles can be incorporated in the Bühlmann-Straub model and the Hachemeister's regression model. For each model, a numeric example is presented.
\end{abstract}

\section{Introduction}

Insurance contracts must be familiar to most people, but it is little known how premiums are estimated. The modern credibility ${ }^{1}$ theory estimates an insured's pure risk premium by striking a balance between the individual's average claims and the overall mean of the claim data. Below is an illustration of this premium estimation technique.

It is well known that an insurance company insures many kinds of risks. These risks are grouped into 'similar risks', called the collective, on the basis of 'objective' risk characteristics [1]. Examples of such risk characteristics are age and sex. Based on the observed data and statistical information, the structure of the collectives could be determined. When setting a premium for a new risk, about which there is no pre-existing claim experience, one could estimate it by the overall mean of the claim data $\bar{X}$, i.e. the average claim amount over all risk collectives. After a period of $n$ years, the aggregate claim amounts observed could contribute to the estimation process. Then the premium for this risk could be estimated by a combination of $\bar{X}$ and the individual average claims, i.e. $\overline{X_{j}}$, the structure is as follows:

$$
z_{j} \bar{X}_{j}+\left(1-z_{j}\right) \bar{X}
$$

A premium such as this is called a credibility premium, $z_{j}$ is the credibility factor, expressing how much faith you can have in the individual average claims. [2].

Bühlmann introduced the balanced Bühlmann model in 1967 and together with Straub

${ }^{*} \mathrm{Li}$ Yang received a bachelor degree in Econometrics \& Operations Research at Maastricht University in 2017, where she currently takes the Research Master in the same field. Contact: li.yang@student.maastrichtuniversity.nl

${ }^{1}$ A premium estimation technique. 
introduced the Bühlmann-Straub model in 1970 which established the theoretical foundation of modern credibility theory. Hachemeister extended these models in Hachemeister's regression model by using linear trend model. In 2013, Georgios Pitselis published a paper illustrating links between credibility theory and quantiles. This improves the premium estimation technique as claim distributions are in general heavy-tailed, and quantile credibility regression detects information about the tail behaviour of claim distributions.

This paper aims to introduce the Bühlmann-Straub model, the Quantile credibility model, Hachemeister's regression model and the Quantile regression credibility model. The rest of the paper is organized as follows. Section 2 covers the concept of quantiles and conditional quantiles. Section 3 discusses quantile regression and its estimation method. The Bühlmann-Straub model will be introduced in section 4, as well as how quantiles are incorporated into this model. Hachemeisters's regression model will be introduced in section 5, as well as how quantiles are incorporated in this model. Section 6 discusses the effect of outliers on these models. Finally, Section 7 draws conclusions.

\section{Quantiles and Conditional Quantiles}

\section{$2.1 \quad$ Definition}

Let $\mathrm{Y}$ be a random variable with the distribution $F_{Y}$ and $\mathrm{p}$ be a real number between 0 and 1, i.e. $0<p<1$. Then the p-quantile $\xi_{p}$ is defined as follows:

$$
\xi_{p}=F_{Y}^{-1}(p)=\inf \left\{y: F_{Y}(y) \geq p\right\}
$$

As the payment could take any value of some entire multiple of the monetary unit, which results in a very large set of possible values, each of them with a very small probability, a continuous $\mathrm{CDF}$ for $F_{Y}$ is more appropriate here. Then the p-quantile of $F_{Y}$ can be obtained by minimizing the following objective function with respect to $\xi_{p}$ :

$$
\begin{aligned}
& p \int_{y \geq \xi_{p}}\left|y-\xi_{p}\right| \mathrm{d} F_{Y}(y)+(1-p) \int_{y<\xi_{p}}\left|y-\xi_{p}\right| \mathrm{d} F_{Y}(y) \\
& =p \int_{y \geq \xi_{p}}\left(y-\xi_{p}\right) \mathrm{d} F_{Y}(y)-(1-p) \int_{y<\xi_{p}}\left(y-\xi_{p}\right) \mathrm{d} F_{Y}(y) .
\end{aligned}
$$

Take the derivative of equation (1) w.r.t. $\xi_{p}$ :

$$
\begin{aligned}
& -p \int_{y \geq \xi_{p}} d F_{Y}(y)+(1-p) \int_{y<\xi_{p}} d F_{Y}(y) \\
& =-p\left[1-F_{Y}\left(\xi_{p}\right)\right]+(1-p) F_{Y}\left(\xi_{p}\right) \\
& =-p+F_{Y}\left(\xi_{p}\right) \\
& \stackrel{\text { set }}{=} 0 .
\end{aligned}
$$

The second derivative $=f_{Y}(y) \geq 0$, which implies that $\xi_{p}=F_{Y}^{-1}(p)$ indeed minimizes the objective function mentioned above.

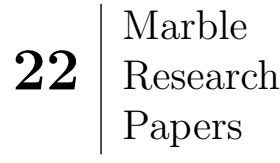


Similarly, in case that $\mathrm{Y}$ has a conditional distribution $F_{Y \mid X}$, the p-quantile is defined as follows:

$$
\xi_{p}=F_{Y \mid X}^{-1}(p)=\inf \left\{y: F_{Y \mid X}(y) \geq p\right\} .
$$

$\xi_{p}$ is a function of $\mathrm{X}$ and minimizes the following objective function:

$$
p \int_{y \geq \xi_{p}}\left|y-\xi_{p}\right| \mathrm{d} F_{Y \mid X}(y)+(1-p) \int_{y<\xi_{p}}\left|y-\xi_{p}\right| \mathrm{d} F_{Y \mid X}(y) .
$$

\subsection{Empirical Quantile Function}

Let $Y_{1}, Y_{2}, \ldots, Y_{n}$ denote the order statistics of $X_{1}, X_{2}, \ldots, X_{n}$, and let $\hat{\xi}_{p}$ denote the sample p-quantile. Assume that the order statistics $Y_{1}, Y_{2}, \ldots, Y_{n}$ partition the support of $\mathrm{X}$ into $\mathrm{n}$ parts and thereby create $\mathrm{n}$ equalling areas under $f(x)$ and above the $\mathrm{X}$-axis, see figure 1 , then each area is on average: $\frac{1}{n}$.

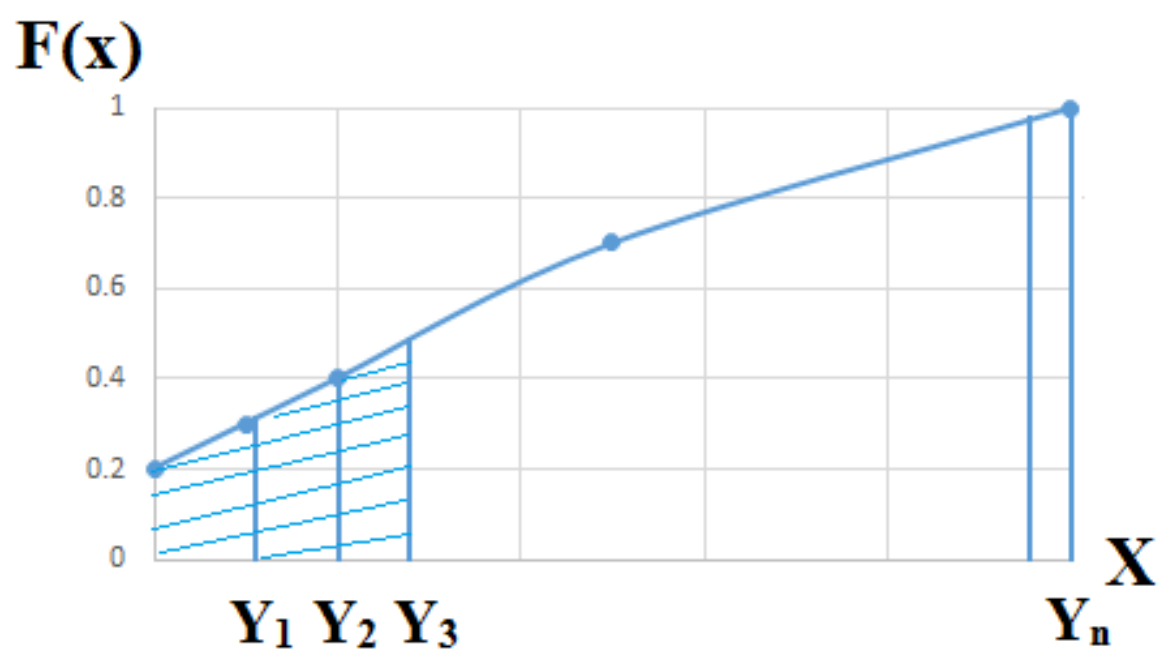

Figure 1: Sample Quantiles

According to the definition, $\mathrm{p}$ is the area under $f(x)$ to the left of $\xi_{p}$. If $\mathrm{np}$ is an integer, $Y_{j}(1 \leq j \leq n)$ serves as an estimator of $\xi_{p}$, namely:

$$
\hat{\xi_{p}}=Y_{j}, \text { if } p=\frac{j}{n} .
$$

In case $j-1<n p<j$, the empirical quantile function can be defined as:

$$
\begin{aligned}
\hat{\xi_{p}} & =Y_{j-1}+\{n p-(j-1)\}\left(Y_{j}-Y_{j-1}\right) \\
& =(j-n p) Y_{j-1}+\left\{n p-(j-1) Y_{j}\right\} .
\end{aligned}
$$

In summary, $\forall$ integer-valued $\mathrm{j} \in[1, n]$,

$$
\hat{\xi}_{p}= \begin{cases}Y_{j} & \text { if } n p=j ; \\ (j-n p) Y_{j-1}+\left\{n p-(j-1) Y_{j}\right\} & \text { if } j-1<n p<j .\end{cases}
$$

Estimating Insurance Premiums using Credibility Theory and Quantiles 


\subsection{Confidence Interval}

This subsection shows how confident that $\xi_{p}$ is contained in the interval, say $\left(Y_{j}, Y_{k}\right)$. Simply, it is to calculate $P\left(Y_{j}<\xi_{p}<Y_{k}\right)$. Let $\mathrm{N}$ denotes the number of $X_{i}$ which is smaller than $\xi_{p}$, then $\mathrm{N}$ is a binomial random variable with $\mathrm{n}$ mutually independent trials and with probability of success $p=P\left(X_{i}<\xi_{p}\right)$. To ensure that $\xi_{p}$ is sandwiched by $Y_{j}$ and $Y_{k}$, the order of $Y_{j}$ must be less than $\xi_{p}$, and the order of $Y_{k}$ greater than $\xi_{p}$, meaning there are at least $\mathrm{j} X_{i}$ s to the left of $\xi_{j}$ and at most (k-1) $X_{i}$ s to the right of $\xi_{j}$, then $j \leq N \leq k-1$. It immediately follows that:

$$
P\left(Y_{j}<\xi_{p}<Y_{k}\right)=\sum_{i=j}^{k-1} P(N=i)=\sum_{i=j}^{k-1}\left(\begin{array}{l}
n \\
i
\end{array}\right)(p)^{i}(1-p)^{n-i}
$$

\section{Quantile Regression}

\subsection{Classical Multiple Linear Regression Model}

To analyse the behaviour of a dependent variable, given a set of explanatory variables, a standard approach is to use multiple linear regression and estimate the parameters by minimizing the sum of squared residuals, which leads to an approximation to the mean function of the conditional distribution of the dependent variable. Below is an illustration of this method.

The form of this regression model for a single observation is as follows:

$$
y_{i}=\beta_{1}+x_{i 2} \beta_{2}+\cdots+x_{i K} \beta_{K}+\epsilon_{i}
$$

If stack all $\mathrm{n}$ observations:

$$
\underbrace{Y}_{\mathrm{n} \times 1}=\underbrace{X}_{\mathrm{n} \times \mathrm{K}} \underbrace{\beta}_{\mathrm{K} \times 1}+\underbrace{\epsilon}_{\mathrm{n} \times 1}
$$

where

$$
\begin{aligned}
& Y=\left(\begin{array}{c}
y_{1} \\
\vdots \\
y_{n}
\end{array}\right), \epsilon=\left(\begin{array}{c}
\epsilon_{1} \\
\vdots \\
\epsilon_{n}
\end{array}\right), \beta=\left(\begin{array}{c}
\beta_{1} \\
\vdots \\
\beta_{K}
\end{array}\right), X=\left(\begin{array}{cc}
1 & x_{1}^{\prime} \\
1 & x_{2}^{\prime} \\
\vdots & \vdots \\
1 & x_{n}^{\prime}
\end{array}\right)=\left(\begin{array}{ccccc}
1 & x_{12} & x_{13} & \cdots & x_{1 K} \\
1 & x_{22} & x_{23} & \cdots & x_{2 K} \\
\vdots & \vdots & \vdots & \ddots & \vdots \\
1 & x_{n 2} & x_{n 3} & \cdots & x_{n K}
\end{array}\right) \\
& \mathbf{2 4} \begin{array}{l}
\text { Marble } \\
\text { Research } \\
\text { Papers }
\end{array}
\end{aligned}
$$


$Y:$ vector of dependent variables, $n$ observations;

$X$ : matrix of $k$ explanatory variables of $n$ observations, with $k \leq n$;

$\beta:$ vector of $k$ unknown parameters;

$\epsilon_{i}$ : assumed to be iid, with $E\left[\epsilon_{i} \mid X\right]=0$, and $\operatorname{Cov}\left[\epsilon_{i}, \epsilon_{j} \mid X\right]=0, \operatorname{Var}\left[\epsilon_{i} \mid X\right]=\sigma^{2}$, let

$\Sigma=\left(\begin{array}{cccc}\epsilon_{1}^{2} \mid X & \epsilon_{1} \epsilon_{2} \mid X & \cdots & \epsilon_{1} \epsilon_{n} \mid X \\ \epsilon_{2} \epsilon_{1} \mid X & \epsilon_{2}^{2} \mid X & \cdots & \epsilon_{2} \epsilon_{n} \mid X \\ \epsilon_{n} \epsilon_{1} \mid X & \epsilon_{n} \epsilon_{2} \mid X & \cdots & \epsilon_{n}^{2} \mid X\end{array}\right)$, then $\Sigma=\left(\begin{array}{cccc}\sigma^{2} & 0 & \cdots & 0 \\ 0 & \sigma^{2} & \cdots & 0 \\ 0 & 0 & \cdots & \sigma^{2}\end{array}\right)$;

the population regression is : $E[Y \mid X]=X \beta$;

the estimate of $E[Y \mid X]: \hat{Y}=X \hat{\beta}$;

the residual : $e=Y-\hat{Y}=Y-X \hat{\beta}$;

the sum of squared residuals: $\sum_{i=1}^{n} e^{\prime} e=\sum_{i=1}^{n}(Y-X \hat{\beta})^{\prime}(Y-X \hat{\beta})$.

The estimated coefficients are calculated as follows:

$$
\begin{aligned}
& \hat{\beta}=\left(X^{\prime} X\right)^{-1}\left(X^{\prime} Y\right), \\
& \text { with } \Sigma_{\hat{\beta}}=\sigma^{2}\left(X^{\prime} X\right)^{-1} .
\end{aligned}
$$

When the assumption $\operatorname{Var}\left[\epsilon_{i} \mid X\right]=\sigma^{2}$ is violated, meaning the value of $\sigma_{i}^{2}$ may differ from each other, namely:

$$
\Sigma=\left(\begin{array}{cccc}
\sigma_{1}^{2} & 0 & \cdots & 0 \\
0 & \sigma_{2}^{2} & \cdots & 0 \\
0 & 0 & \cdots & \sigma_{n}^{2}
\end{array}\right)
$$

the method of weighted least squares is used, then

$$
\begin{aligned}
& \hat{\beta}=\left(X^{\prime} \Sigma^{-1} X\right)^{-1}\left(X^{\prime} \Sigma^{-1} Y\right), \\
& \text { with } \Sigma_{\hat{\beta}}=\sigma^{2}\left(X^{\prime} \Sigma^{-1} X\right)^{-1} .
\end{aligned}
$$

Weighted least squares estimation is used in the Hachemeister's regression model which will be covered in later section.[4]

\subsection{OLS vs Quantile}

The mean measure, obtained from the classical multiple linear regression, represents the average behaviour of a distribution, but provides little information about the tail behaviour of that distribution. In 1918, Koenker and Bassett proposed the quantile regression, which enables to estimate various quantile functions of a conditional distribution. This approach provides a more comprehensive picture of the effect of dependent variables on independent variable by putting different quantile regressions together in one graph. To see the difference two simple examples are presented. Both disturbances are normally distributed, data of figure $2 a$ is with constant variance, data of figure $2 b$ is with non-constant variance. As can be seen from figure $2 b$ that the dependent variable becomes more variable when the independent variable increases.

Estimating Insurance Premiums using Credibility Theory and Quantiles 


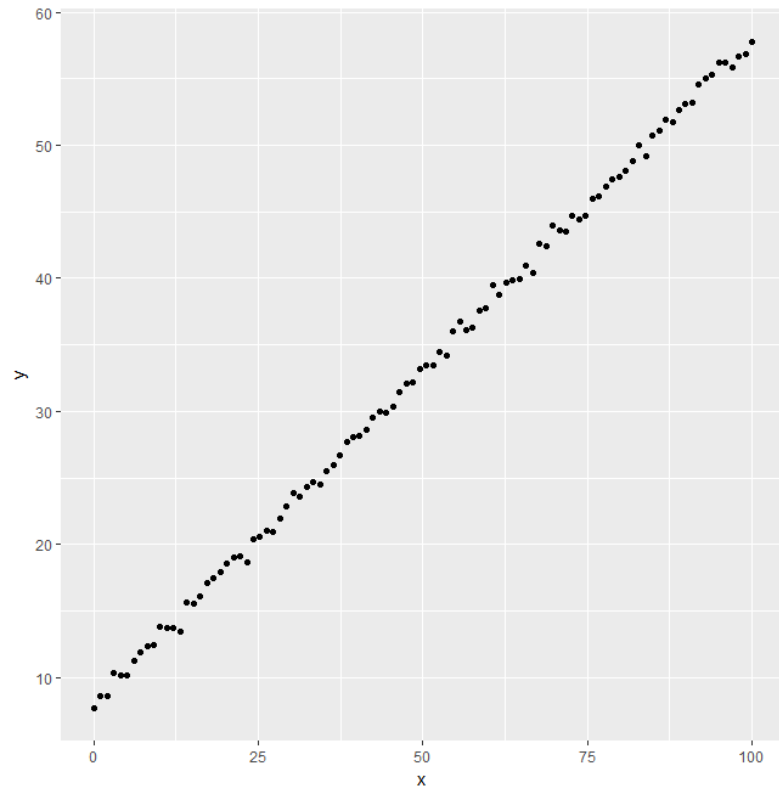

(A) Data (constant variance)

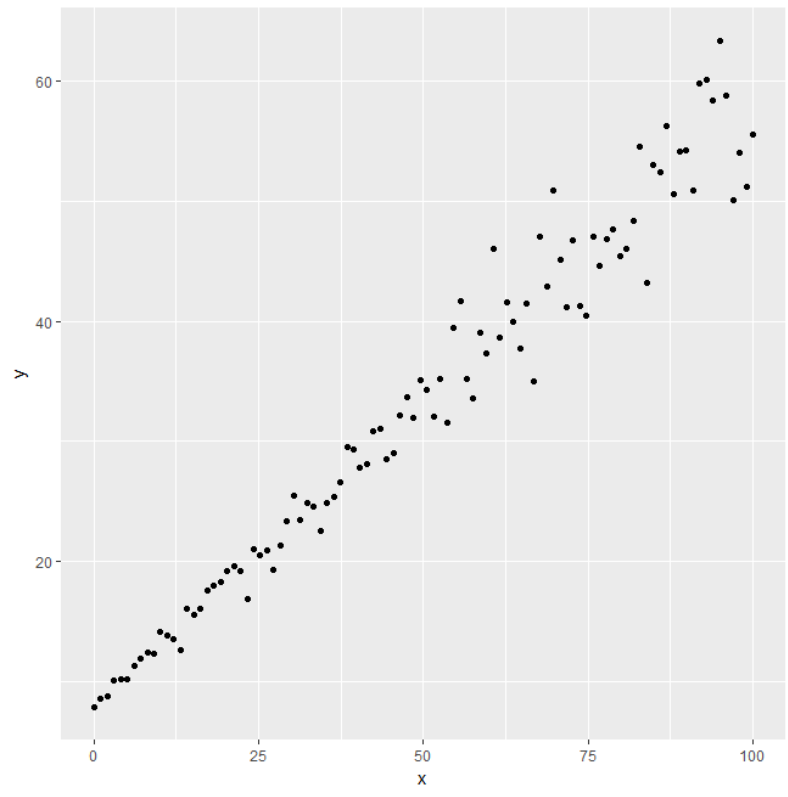

(B) Data (non-constant variance)

Figure 2: Two simple data sets

These two data sets are generated by employing the following R-codes :

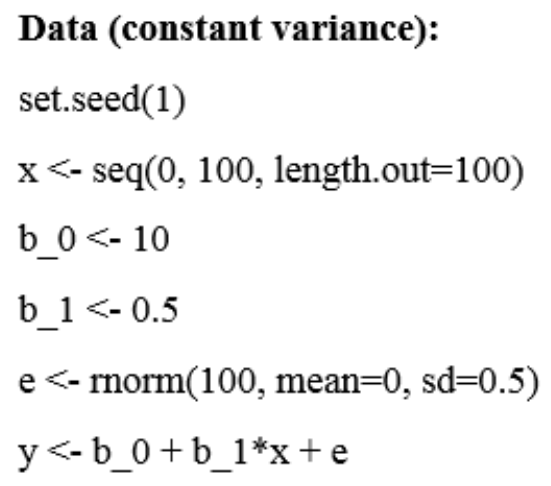

$y<-b+0+b$

\section{Data (non-constant variance):}

$$
\begin{aligned}
& \text { set.seed(1) } \\
& x<-\operatorname{seq}(0,100, \text { length.out }=100) \\
& \text { b_0 }<-10 \\
& \text { b_1 }<-0.5 \\
& \text { sig }<-1+0.2^{*} x \\
& \text { e }<- \text { rnorm }(100, \text { mean }=0, \text { sd }=\text { sig }) \\
& y<- \text { b_0 }+ \text { b_ } 1 * x+e
\end{aligned}
$$

Figure 3: Two artificial data

First, apply OLS regression to both data sets. Figure $4 a$ and figure $4 b$ show the results separately. When the variance is constant, the conditional mean from OLS regression provides a good estimate of dependent variable. However, it is not the case when the variance is not constant, as the conditional mean becomes less meaningful when the explanatory variable increases, seen from figure $4 b$.

$26 \begin{aligned} & \text { Marble } \\ & \text { Research } \\ & \text { Papers }\end{aligned}$ 


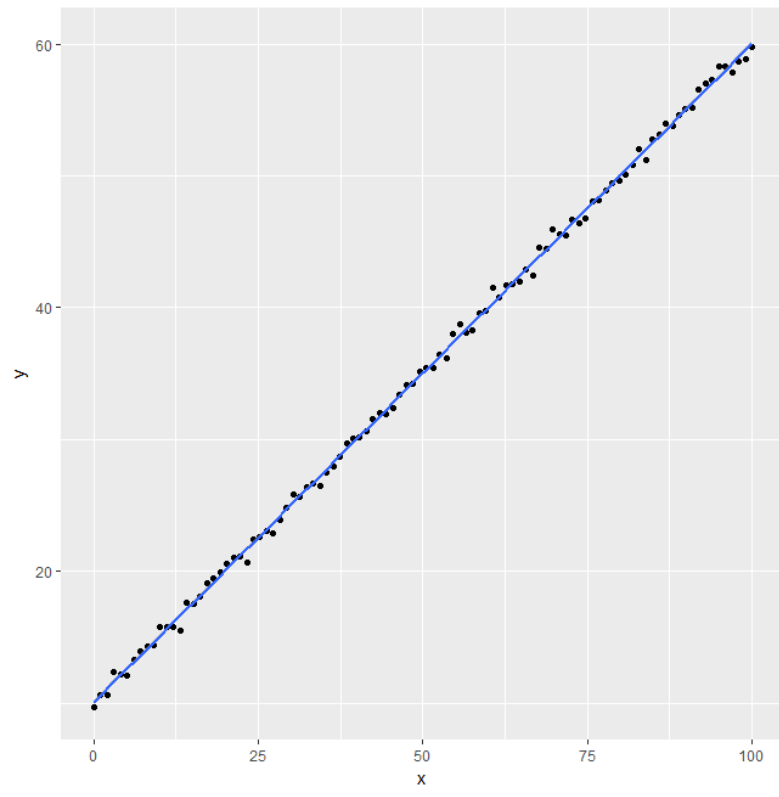

(A) OLS (constant variance)

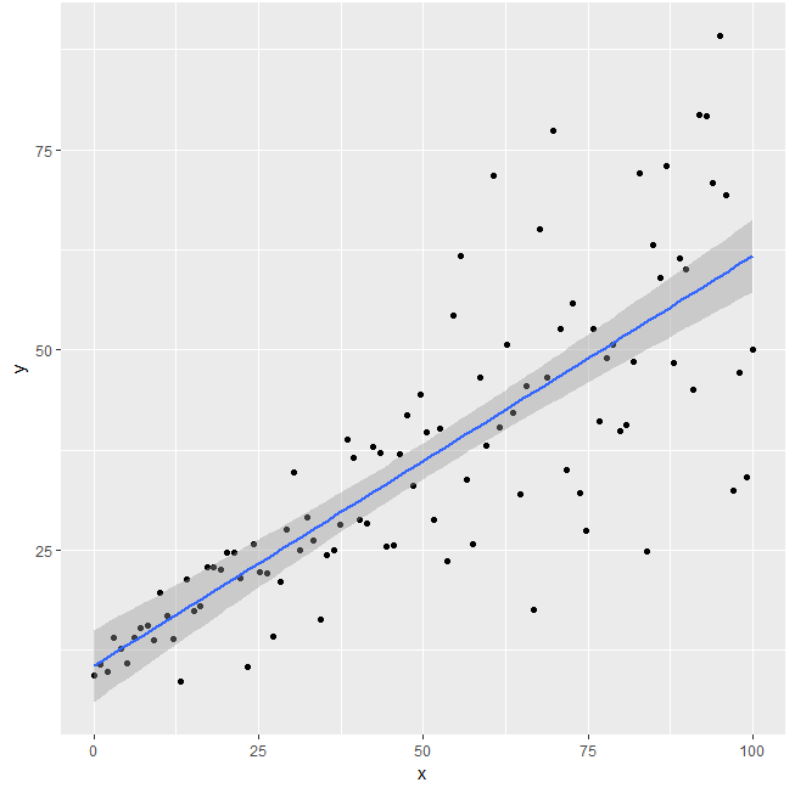

(в) OLS (non-constant variance)

FiguRE 4: OLS regression

Second, apply quantile regression to both data sets. The slope coefficient for the quantile indicated on the $\mathrm{x}$-axis for both data sets are shown separately in figure $5 a$ and figure $5 b$. The horizontal solid lines are the OLS coefficient estimates, the horizontal dotted lines are its confidence interval; the black curved lines are the quantile coefficient estimates, the shaded area is its confidence interval.

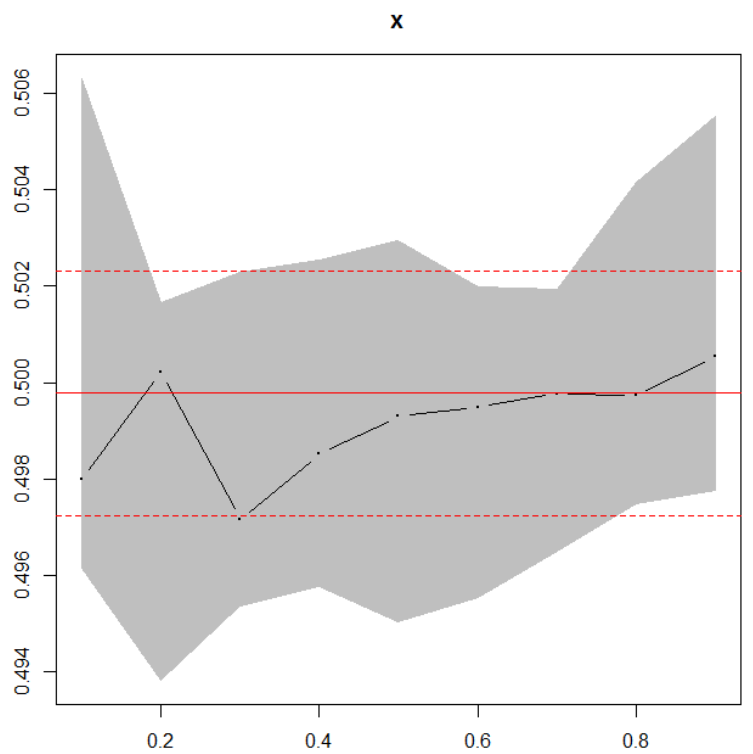

(A) Quantile coefficients (constant variance)

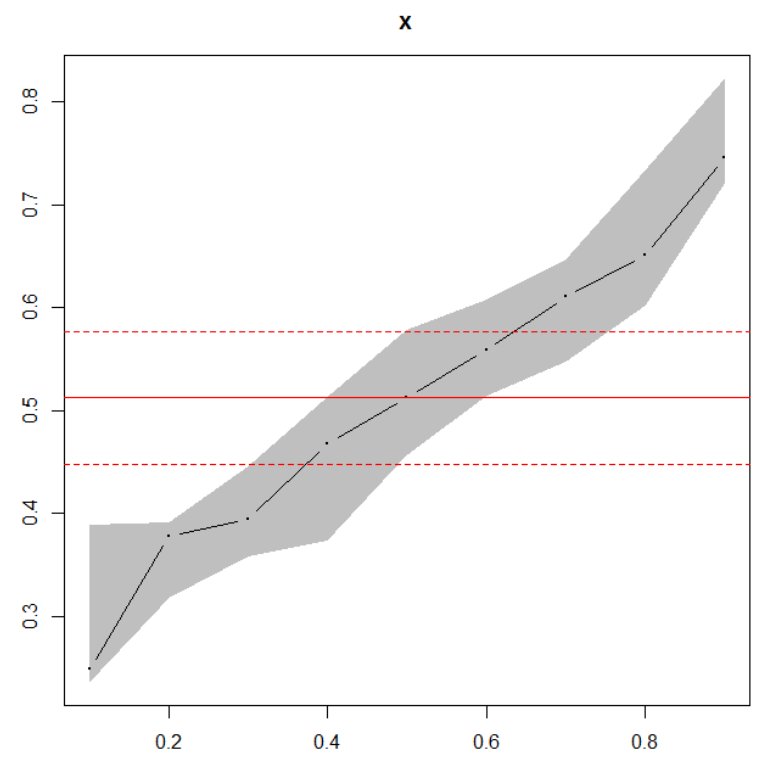

(в) Quantile coefficients (non-constant variance)

FiguRE 5: Quantile regression

Estimating Insurance Premiums using Credibility 
Figure $5 a$ shows that the quantile coefficient estimates fall well inside the confidence interval when the variance is constant, meaning the quantile estimates are not significantly different from the OLS coefficient estimates. On the other hand, when the variance is not constant, figure $5 b$ shows that the majority of quantile coefficient estimates fall outside of the OLS confidence interval, meaning quantile coefficient estimates are significantly different from OLS coefficient estimates. This implies that, under the assumption that the disturbance is normally distributed and with constant variance, there is no efficiency gain by using quantile regression; but quantile estimations perform better when the variance is not constant, as the coefficients can vary across quantiles. To point out, the estimated coefficients are significantly different from 0 , when 0 is not in the confidence interval. In case the error term is not normally distributed, quantile regression may be more efficient than the least squares estimation, mentioned in Pitselis' paper.

\subsection{The Method of Quantile Regression}

Similar to general linear regression model, quantile regression model has the following regression equation:

$$
y_{p i}=x_{i}^{\prime} \beta_{p}+u_{p i} .
$$

Here, $y_{p i}$ is the p-quantile of the observed risks $y_{i 1}, \ldots, y_{i n} ; \beta_{p}$ is the corresponding regression coefficients. As p can take any value between 0 and $1, \beta_{p}$ may have different value for each choice of $\mathrm{p}$, which is different from the linear regression model where $\beta_{p}$ is a fixed parameter. The p-conditional quantile of $y_{i}$ given $x_{i}$ is:

$$
Q_{p}\left(y_{i} \mid x_{i}\right)=x_{i}^{\prime} \beta_{p}
$$

In view of (1), $Q_{p}$ of quantile regression can be written in the following form:

$$
Q_{p}\left(y_{i} \mid x_{i}\right)=p \int_{y_{i} \geq x_{i}^{\prime} \beta_{p}}\left|y_{i}-x_{i}^{\prime} \beta_{p}\right| \mathrm{d} F_{Y \mid X}(y)+(1-p) \int_{y_{i}<x_{i}^{\prime} \beta_{p}}\left|y_{i}-x_{i}^{\prime} \beta_{p}\right| \mathrm{d} F_{Y \mid X}(y)
$$

The estimator of $\beta_{p}$ can be obtained by minimizing its sample counterpart:

$$
\begin{aligned}
\hat{Q_{p}}\left(y_{i} \mid x_{i}\right) & =\frac{1}{N}\left[p \sum_{i: y_{i} \geq x_{i}^{\prime} \beta_{p}}^{N}\left|y_{i}-x_{i}^{\prime} \beta_{p}\right|+(1-p) \sum_{i: y_{i}<x_{i}^{\prime} \beta_{p}}^{N}\left|y_{i}-x_{i}^{\prime} \beta_{p}\right|\right] \\
& =\frac{1}{N} \sum_{i=1}^{N} g\left(y_{i}-x_{i}^{\prime} \beta_{p} \mid p\right)
\end{aligned}
$$

where

$$
g\left(y_{i}-x_{i}^{\prime} \beta_{p} \mid p\right)= \begin{cases}p\left(y_{i}-x_{i}^{\prime} \beta_{p}\right) & \text { if } y_{i}-x_{i}^{\prime} \beta_{p} \geq 0 \\ (1-p)\left(y_{i}-x_{i}^{\prime} \beta_{p}\right) & \text { if } y_{i}-x_{i}^{\prime} \beta_{p}<0\end{cases}
$$

For $p=0.5$,

$$
2 \hat{Q}_{0.5}\left(y_{i} \mid x_{i}\right)=\frac{1}{N} \sum_{i=1}^{n}\left|y_{i}-x_{i}^{\prime} \beta_{p}\right|
$$

This is a regression estimated via the method of LAD: Least Absolute Deviations Estimation, referred to as a "median regression". This minimization problem can be set up as

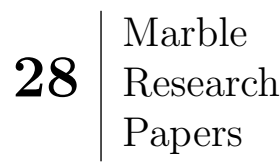


a linear programming problem, and quantile regression can be implemented in software such as R.

The function $g\left(y_{i}-x_{i}^{\prime} \beta_{p} \mid p\right)$ is known as the "check function": piecewise linear and not differentiable at $y_{i}=x_{i}^{\prime} \beta_{p}$. One way to minimize $\hat{Q}_{p}\left(y_{i} \mid x_{i}\right)$ is to use the directional derivatives of $\hat{Q}_{p}\left(y_{i} \mid x_{i}\right)$. The directional derivatives works as follows:

let $\omega$ be an arbitrary direction,

$$
\begin{aligned}
\left.\frac{d}{d \delta} \hat{Q}_{p}\left(\beta_{p}+\delta \omega ; p\right)\right|_{\delta=0} & =\left.\frac{1}{N}\left(\frac{d}{d \delta} \sum_{i=1}^{N}\left(\xi_{p}-x_{i}^{\prime} \beta_{p}-\delta x_{i}^{\prime} \omega\right)\left(p-I_{\left\{\xi_{p}-x_{i}^{\prime} \beta_{p}-\delta x_{i}^{\prime} \omega<0\right\}}\right)\right)\right|_{\delta=0} \\
& =-\frac{1}{N} \sum_{i=1}^{N} \psi_{p}\left(\xi_{p}-x_{i}^{\prime} \beta_{p},-x_{i}^{\prime} \omega\right) x_{i}^{\prime} \omega
\end{aligned}
$$

where

$$
\psi_{p}\left(\xi_{p}-x_{i}^{\prime} \beta_{p},-x_{i}^{\prime} \omega\right)= \begin{cases}p-I_{\left\{\xi_{p}-x_{i}^{\prime} \beta_{p}<0\right\}} & \text { if } \xi_{p}-x_{i}^{\prime} \beta_{p} \neq 0 \\ p-I_{\left\{-x_{i}^{\prime} \omega<0\right\}} & \text { if } \xi_{p}-x_{i}^{\prime} \beta_{p}=0\end{cases}
$$

To determine the quantile regression estimator of $\beta_{p}$ is to find a point that minimizes the function $Q_{p}\left(\beta_{p} ; p\right)$, which is equal to find a point where $\frac{d}{d \delta} Q_{p}\left(\beta_{p}+\delta \omega ; p\right)$ is nonnegative at all directions. The p-quantile regression estimator of $\beta_{p}$ is denoted as $\hat{\beta}_{p}$, quantile regression residuals are $\hat{e}_{i}(p)=\xi_{p}-x_{i}^{\prime} \hat{\beta}_{p}$.

If $\operatorname{plim} \frac{1}{n} X^{\prime} X$ equals a finite and positive definite matrix, meaning data is wellbehaved, $\hat{\beta}_{p}$ is consistent and asymptotically normally distributed with asymptotic covariance matrix

$$
\text { Asy.Var }\left[\hat{\beta}_{p}\right]=\frac{1}{n} H^{\prime} G H^{\prime},
$$

where

$$
\begin{aligned}
& H=p l i m \frac{1}{n} \sum_{i=1}^{n} f_{p}\left(0 \mid x_{i}\right) x_{i} x_{i}^{\prime}, \\
& G=p \lim \frac{p(-p)}{n} \sum_{i=1}^{n} x_{i} x_{i}^{\prime} .
\end{aligned}
$$

It is worth mentioning that computation of $f_{p}\left(0 \mid x_{i}\right)$ could be complicated.[4]

\section{Credibility Models}

\subsection{The Bühlmann-Straub model}

Let $X_{j t}$ be a claim amount for contract $\mathrm{j}$ at time t, then $X_{j t}$ can be decomposed into three parts:

$$
X_{j t}=m+\Xi_{j}+\Xi_{j t}, \quad j=1, \ldots, J, t=1, \ldots, T+1
$$

m: the overall mean claim, i.e. the expected value of the claim amount for an arbitrary policyholder in the portfolio; 
$\Xi_{j}$ : denotes a random deviation from $\mathrm{m}$, iid, with $E\left[\Xi_{j}\right]=0, \operatorname{Var}\left[\Xi_{j}\right]=a$;

$\Xi_{j t}$ : denotes the deviation for year t from the long-term average. $\Xi_{j 1}, \Xi_{j 2}, \ldots$ are iid, with $E\left[\Xi_{j t}\right]=0, \operatorname{Var}\left[\Xi_{j t}\right]=s^{2} / w_{j t}, w_{j t}$ is the weight attached to observation $X_{j t}$.

This model assumes equal numbers of policies in $X_{j t}$.

Then the unbiased predictor of $X_{j, n+1}$ which minimizes the following objective function :

$$
E\left[\left\{m+\Xi_{j}-\sum_{j=1}^{K} \sum_{i=1}^{n} h_{j i} X_{j i}\right\}^{2}\right], \text { subject to } E\left[m+\Xi_{j}\right]=\sum_{i, t} h_{j i} X_{j i}
$$

equals the credibility premium:

$$
z_{j} X_{j w}+\left(1-z_{j}\right) X_{z w}
$$

where

$$
\begin{aligned}
& z_{j}=\frac{a w_{j \Sigma}}{s^{2}+a w_{j \Sigma}} ; z_{\Sigma}=\sum_{j=1}^{J} z_{j} ; \\
& w_{j \Sigma}=\sum_{t=1}^{T} w_{j t} ; w_{\Sigma \Sigma}=\sum_{j=1}^{J} w_{j \Sigma} ; \\
& X_{j w}=\sum_{t=1}^{T} \frac{w_{j t}}{w_{j \Sigma}} X_{j t} ; X_{w w}=\sum_{j=1}^{J} \frac{w_{j \Sigma}}{w_{\Sigma \Sigma}} X_{j w} ; X_{z w}=\sum_{j=1}^{J} \frac{z_{j}}{z_{\Sigma}} X_{j w} .
\end{aligned}
$$

Unbiased parameter estimates:

$$
\begin{aligned}
& \widetilde{m}=X_{w w} ; \\
& \widetilde{s^{2}}=\frac{1}{J(T-1)} \sum_{j, t} w_{j t}\left(X_{j t}-X_{j w}\right)^{2} ; \\
& \widetilde{a}=\frac{\sum_{j} w_{j \Sigma}\left(X_{j w}-X_{w w}\right)^{2}-(J-1) \widetilde{s^{2}}}{w_{\Sigma \Sigma}-\sum_{j} w_{j \Sigma}^{2} / w_{\Sigma \Sigma}}, \text { in case } \tilde{a}=0, \text { set } \tilde{a}=(J-1) \times \tilde{s}^{2} .
\end{aligned}
$$

It is worth noting that all the equations hold if given $\Xi_{j}, \Xi_{j t}$ is iid and with $E\left[\Xi_{j t} \mid \Xi_{j}\right]=0$. Then $\operatorname{Cov}\left[\Xi_{j t}, \Xi_{j u}\right]=0(t \neq u), \operatorname{Cov}\left[\Xi_{j}, \Xi_{j t}\right]=0$, which means that $\Xi_{j t}$ and $\Xi_{j u}$ are uncorrelated, $\Xi_{j}$ and $\Xi_{j t}$ are uncorrelated, but the random variables $X_{j t}$ are not marginally uncorrelated.

In the Quantile credibility model and Quantile regression credibility model Georgios Pitselis uses $\Theta_{j}$ instead of $\Xi_{j}$, and assumes that given $\Theta_{j}, X_{1 j}, \ldots, X_{n j}$ are conditionally independent and with the same distribution function.[2]

$\mathbf{3 0} \mid \begin{aligned} & \text { Marble } \\ & \text { Research } \\ & \text { Papers }\end{aligned}$ 


\section{Numeric Example}

Apply the Bühlmann-Straub model to Hachemeister's claims data set (the severity average loss per claim per state in 12 periods). The data set is shown below.

\begin{tabular}{l|ccccc|ccccc}
\hline \multirow{2}{*}{$\mathrm{P}$} & \multicolumn{5}{|c|}{ Average loss per claim } & \multicolumn{5}{c}{ Number of claims per period } \\
\cline { 2 - 12 } & State1 & State2 & State3 & State4 & State5 & State1 & State2 & State13 & State4 & State5 \\
\hline 1 & 1738 & 1364 & 1759 & 1223 & 1456 & 7861 & 1622 & 1147 & 407 & 2902 \\
\hline 2 & 1642 & 1408 & 1685 & 1146 & 1499 & 9251 & 1742 & 1357 & 396 & 3172 \\
\hline 3 & 1794 & 1597 & 1479 & 1010 & 1609 & 8706 & 1523 & 1329 & 348 & 3046 \\
\hline 4 & 2051 & 1444 & 1763 & 1257 & 1741 & 8575 & 1515 & 1204 & 341 & 3068 \\
\hline 5 & 2079 & 1342 & 1674 & 1426 & 1482 & 7917 & 1622 & 998 & 315 & 2693 \\
\hline 6 & 2234 & 1675 & 2103 & 1532 & 1572 & 8263 & 1602 & 1077 & 328 & 2901 \\
\hline 7 & 2032 & 1470 & 1502 & 1953 & 1606 & 9456 & 1964 & 1277 & 352 & 3275 \\
\hline 8 & 2035 & 1448 & 1622 & 1123 & 1735 & 8003 & 1515 & 1218 & 331 & 2697 \\
\hline 9 & 2115 & 1464 & 1828 & 1343 & 1607 & 7365 & 1527 & 896 & 287 & 2663 \\
\hline 10 & 2262 & 1831 & 2155 & 1243 & 1573 & 7832 & 1748 & 1003 & 384 & 3017 \\
\hline 11 & 2267 & 1612 & 2233 & 1762 & 1613 & 7849 & 1654 & 1108 & 321 & 3242 \\
\hline 12 & 2517 & 1471 & 2059 & 1306 & 1690 & 9077 & 1861 & 1121 & 342 & 3425 \\
\hline
\end{tabular}

TABLE 1: Hachemeister's claims data set.

Calculations:

$$
\begin{aligned}
& w_{1 \Sigma}=100155, w_{2 \Sigma}=19895, w_{3 \Sigma}=13735, w_{4 \Sigma}=4152, w_{5 \Sigma}=36110 \\
& w_{\Sigma \Sigma}=174047 ; \\
& X_{1 w}=2060.921, X_{2 w}=1511.224, X_{3 w}=1805.843, X_{4 w}=1352.976, X_{5 w}=1599.829 ; \\
& X_{w w}=1865.404 ; \\
& \widetilde{s^{2}}=139120026, \widetilde{a}=89638.73 ; \\
& Z_{1}=0.9847404, Z_{2}=0.9276352, Z_{3}=0.8984754, Z_{4}=0.7279092, Z_{5}=0.9587911 . \\
& Z_{\Sigma}=4.497551, X_{z w}=1683.713 .
\end{aligned}
$$

Premiums estimated:

\begin{tabular}{c|c|c|c|c|c}
\hline & state1 & state2 & state3 & state4 & state5 \\
\hline premium & 2055.165 & 1523.706 & 1793.444 & 1442.967 & 1603.285 \\
\hline
\end{tabular}

TABLE 2: premiums Bühlmann-Straub model

\subsection{Quantile Credibility Model}

Based on Bülmann's classical model assumptions, Georgios Pitselis developed the quantile credibility model. Let $X_{j 1}, X_{j 2}, \ldots, X_{j n_{j}}$ be the observed total claim amounts (or the total number of claims) in period $\mathrm{i}=1,2, . ., n_{j}$ for contract $\mathrm{j}=1,2, \ldots, \mathrm{K}$, let $\Theta_{j}$ be an unobservable risk parameter that describes for contract $\mathrm{j}$, and $\hat{\xi}_{p j}$ be the estimator of $\xi_{p j}$.

Estimating Insurance Premiums using Credibility Theory and Quantiles 
The model assumptions:

(i) Given $\Theta_{j}=\theta_{j}$, the observations $X_{j 1}, \ldots, X_{j n_{j}}$ are conditionally independent with the same distribution function;

(ii) $\Theta_{j}$ is a random variable with distribution $\mathrm{U}$;

(iii) $\Xi_{p}\left(\Theta_{j}\right)=E\left(\hat{\xi}_{p j} \mid \Theta_{j}\right)$;

(iv) $\nu_{p}=\operatorname{Var}\left(\hat{\xi}_{p j} \mid \Theta_{j}\right)$.

The structural parameters are defined as follows:

$\Xi_{p}=E\left[\Xi_{p}\left(\Theta_{j}\right)\right], \quad s_{\xi_{p}}^{2}=E\left[\nu_{p}\left(\Theta_{j}\right)\right], \quad \psi_{p}=\operatorname{Var}\left[\Xi_{p}\left(\Theta_{j}\right)\right]$.

Then the linear quantile credibility estimation with $\mathrm{K}$ contracts can be defined as:

$$
\Xi_{p}^{C r e d}=Z_{p j} \hat{\xi}_{p}+\left(1-Z_{p j}\right) \Xi_{p}
$$

where

$$
Z_{p j}=\frac{\psi_{p}}{E\left[\nu_{p}\left(\Theta_{j}\right)\right]+\psi_{p}}
$$

Proof Define a linear Bayes estimator of $\xi_{p}$ as $g_{j}\left(c_{0}^{j}, c_{p l}^{j}, \hat{\xi}_{l p}\right)=c_{0}^{j}+\sum_{l=1}^{K} c_{l p}^{j} \hat{\xi}_{l p}$. The best estimator $g\left(X_{j}\right)$ minimizes the following objective function:

$$
Q=E\left[\Xi_{p}\left(\Theta_{j}\right)-c_{0}^{j}-\sum_{l=1}^{K} c_{l p}^{j} \hat{\xi}_{l p}\right]^{2}
$$

Taking the derivative of (4) with respect to $c_{0}^{j}, c_{l p}^{j}{ }^{2}$, respectively

$$
\left\{\begin{array}{l}
\frac{d Q}{d c_{0}^{j}}=2 E\left[\left(\Xi_{p}\left(\Theta_{j}\right)-c_{0}^{j}-\sum_{l=1}^{K} c_{l p}^{j} \hat{\xi}_{l p}\right)(-1)\right] \stackrel{\text { set }}{=} 0, \\
\frac{d Q}{d c_{l^{\prime} p}^{j}}=2 E\left[\left(\Xi_{p}\left(\Theta_{j}\right)-c_{0}^{j}-\sum_{l=1}^{K} c_{l p}^{j} \hat{\xi}_{l p}\right)\left(-\hat{\xi}_{l^{\prime} p}\right)\right] \stackrel{\text { set }}{=} 0 .
\end{array}\right.
$$

Then

$$
\left\{\begin{array}{l}
E\left[\Xi_{p}\left(\Theta_{j}\right)\right]-c_{0}^{j}-\sum_{l=1}^{K} c_{l p}^{j} E\left(\hat{\xi}_{l p}\right)=0, \quad(\star) \\
E\left[\Xi_{p}\left(\Theta_{j}\right) \hat{\xi}_{l^{\prime} p}\right]-c_{0}^{j} E\left(\hat{\xi}_{l^{\prime} p}\right)-E\left(\hat{\xi}_{l^{\prime} p}\right) \sum_{l=1}^{K} c_{l p}^{j} E\left(\hat{\xi}_{l p}\right)=0 .
\end{array}\right.
$$

Multiply the equation $(\star)$ by $E\left(\hat{\xi}_{l^{\prime} p}\right)$ :

$$
\left\{\begin{array}{l}
E\left[\Xi_{p}\left(\Theta_{j}\right)\right] E\left[\hat{\xi}_{l^{\prime} p}\right]-c_{0}^{j} E\left(\hat{\xi_{l p}}\right)-\sum_{l=1}^{K} c_{l p}^{j} E\left(\hat{\xi_{p l}}\right) E\left[\hat{\xi}_{l^{\prime} p}\right]=0, \\
E\left[\Xi_{p}\left(\Theta_{j}\right) \hat{\xi}_{l^{\prime} p}\right]-c_{0}^{j} E\left(\hat{\xi}_{l^{\prime} p}\right)-E\left[\hat{\xi}_{l^{\prime} p}\right] \sum_{l=1}^{K} c_{l p}^{j} E\left(\hat{\xi}_{l p}\right)=0 .
\end{array}\right.
$$

\footnotetext{
${ }^{2}$ In Pitselis' paper, he uses $p^{\prime}$ insteas of $\mathrm{p}$, which could be a typo.
} 
It immediately follows that:

$$
\begin{gathered}
E\left[\Xi_{p}\left(\Theta_{j}\right)\right] E\left[\hat{\xi}_{l^{\prime} p}\right]-E\left[\Xi_{p}\left(\Theta_{j}\right) \hat{\xi}_{l^{\prime} p}\right]=E\left[\hat{\xi}_{l^{\prime} p}\right] \sum_{l=1}^{K} c_{l p}^{j} E\left(\hat{\xi}_{l p}\right)-\sum_{l=1}^{K} c_{l p}^{j} E\left(\hat{\xi_{p l}}\right) E\left[\hat{\xi}_{l^{\prime} p}\right] \\
\operatorname{Cov}\left[\Xi_{p}\left(\Theta_{j}\right), \hat{\xi}_{l^{\prime} p}\right]=\sum_{l=1}^{K} c_{l p}^{j} \operatorname{Cov}\left[\hat{\xi}_{l p}, \hat{\xi}_{l^{\prime} p}\right] .
\end{gathered}
$$

Therefore, if $l^{\prime}=j$ :

$$
\begin{aligned}
\operatorname{Cov}\left[\Xi_{p}\left(\Theta_{j}\right), \hat{\xi}_{j p}\right] & =c_{j p} \operatorname{Var}\left[\hat{\xi}_{j p}\right] \\
c_{j p} & =\frac{\operatorname{Cov}\left[\Xi_{p}\left(\Theta_{j}\right), \hat{\xi}_{j p}\right]}{\operatorname{Var}\left[\hat{\xi}_{j p}\right]} \\
& =\frac{E\left[\operatorname{Cov}\left[\Xi_{p}\left(\Theta_{j}\right), \hat{\xi}_{j p} \mid \Theta_{j}\right]\right]+\operatorname{Cov}\left[E\left[\Xi_{p}\left(\Theta_{j}\right) \mid \Theta_{j}\right], E\left[\hat{\xi}_{j p}\left(\Theta_{j}\right) \mid \Theta_{j}\right]\right]}{E\left[\operatorname{Var}\left(\hat{\xi}_{j p} \mid \Theta_{j}\right)\right]+\operatorname{Var}\left[E\left(\hat{\xi}_{j p} \mid \Theta_{j}\right)\right]} \\
& =\frac{\operatorname{Var}\left[\Xi_{p}\left(\Theta_{j}\right)\right]}{E\left[\nu_{p}\left(\Theta_{j}\right)\right]+\operatorname{Var}\left[\Xi_{p}\left(\Theta_{j}\right)\right]} \\
& =\frac{\psi_{p}}{E\left[\nu_{p}\left(\Theta_{j}\right)\right]+\psi_{p}} .
\end{aligned}
$$

\section{Parameter estimation:}

$$
\begin{aligned}
& \widehat{\Xi_{p}}=\bar{\xi}_{p .}=\frac{1}{K} \sum_{j=1}^{K} \hat{\xi}_{j p}, \\
& E\left[\widehat{\nu_{p}\left(\Theta_{j}\right)}\right]=\frac{1}{K} \sum_{j=1}^{K} \frac{\hat{\omega}_{p}\left(\Theta_{j}\right)}{n_{j}}, \\
& \hat{\psi}_{p}=\frac{1}{K-1} \sum_{j=1}^{K}\left(\hat{\xi}_{j p}-\bar{\xi}_{p .}\right)^{2}-\frac{1}{K} \sum_{j=1}^{K} \frac{\hat{\omega}_{p}\left(\Theta_{j}\right)}{n_{j}} .
\end{aligned}
$$

Then the linear quantile credibility with $\mathrm{K}$ contracts can be estimated as:

$$
{\widehat{\Xi_{j p}}}^{\text {Cred }}=\widehat{Z}_{j p} \hat{\xi}_{j p}+\left(1-\widehat{Z}_{j p}\right) \widehat{\Xi}_{p}
$$

where

$$
\begin{aligned}
& Z_{j p}=\frac{\widehat{\psi_{p}}}{E\left[\widehat{\nu_{p}\left(\Theta_{j}\right)}\right]+\widehat{\psi_{p}}}, \\
& \widehat{\omega_{p}}(\Theta)=\frac{n^{2}\left(y_{\lfloor n p+l\rfloor}-y_{\lfloor n p-l\rfloor}\right)^{2}}{4 Z_{1-\alpha / 2}^{2}} \\
& l=Z_{1-\alpha / 2} \sqrt{n p(1-p)}
\end{aligned}
$$

Note that $\lfloor *\rfloor$ is the integer part of $*, \alpha$ is the significance level.

Estimating Insurance Premiums using Credibility 


\section{Numeric Example}

Apply this method to Hachemeister's claims data set. Here only premiums for the median $(\mathrm{p}=0.5)$ are presented. $\alpha$ is chosen to be 0.05 , then $l=3.394757$. A shortcoming of this approach, however, if $\mathrm{p}$ is below 0.366 (approximately), $y_{\lfloor n p-l\rfloor}$ will be invalid, as $0<n p-l<1$ because of small sample size, whereas order statistic starts at $y_{1}$.

Premiums estimated:

\begin{tabular}{c|c|c|c|c|c}
\hline & state1 & state2 & state3 & state4 & state5 \\
\hline premium & 1521.66 & 1681.396 & 1602.864 & 1730.946 & 1644.133 \\
\hline
\end{tabular}

TABLE 3: Premiums Quantile credibility model

\section{Regression Credibility Models}

\subsection{Hachemeister's Regression Model}

Hachemeister forecasted average claim amounts for bodily injury claims classified by state in the USA. The claim data consists 12 periods, ranges from the third quarter of 1970 to the second quarter of 1973. Due to inflation these data is affected by time trends. In his model he regressed the claims averages for each state on explanatory variables: constant and trend(1:12), constructed a diagonal weighting matrix by putting claim frequencies in the diagonal, computed the regression coefficients using weighted least squares estimation. He estimated the credibility factor for the individual regression coefficients, then set premium as the predicted claims average for the next period.

The model is as follows:

let $Y_{j}=\left(Y_{j 1}, Y_{j 2}, \cdots, Y_{j n}\right)^{\prime}$ be an observation vector of the $j t h$ risk, in Hachemeister's regression model the entries are the claims averages of state $\mathrm{j}$ in quarter $\mathrm{t}$; let $w_{j}=\left(\omega_{j 1}, \omega_{j 2}, \cdots, \omega_{i n}\right)^{\prime}$ be the associated known weights, in this model $\omega_{j t}$ are the corresponding number of claims; let $\Theta_{j}$ be the set of all potential and possible values of the risk profile $\theta_{j}$ in the portfolio.

This model assumes that given $\Theta_{j}, X_{j}$ satisfies the regression equation:

$$
Y_{j}=X_{j} \beta_{j}+\epsilon_{j}
$$

The model assumptions:

(i) $\Theta_{1}, \Theta_{2}, \cdots$ are identically distributed, the pairs $\left(\Theta_{1}, Y_{1}\right), \cdots$ are independent;

(ii)Given $\Theta_{j}, Y_{j 1}, Y_{j 2}, \cdots$, are independent, and

$$
\underbrace{E\left[Y_{j} \mid \Theta_{j}\right]}_{\mathrm{n} \times 1}=\underbrace{X_{j}}_{\mathrm{n} \times \mathrm{K}} \underbrace{\beta\left(\Theta_{j}\right)}_{\mathrm{K} \times 1}
$$

where

$\beta\left(\Theta_{j}\right)=$ vector of unknown coef fients of length $K(K \leq n)$, is considered as a random variable, its distribution is determined by the sturcture of the collective, $X_{j}=$ known fixed matrix of rank $K$;

$34 \mid \begin{aligned} & \text { Marble } \\ & \text { Research } \\ & \text { Papers }\end{aligned}$


(iii) $\operatorname{Cov}\left(X_{j}, X_{j}^{\prime} \mid \Theta_{j}\right)=\sigma^{2}\left(\Theta_{j}\right) W_{j}^{-1}$.

The structural parameters:

$s^{2}=E\left[\sigma^{2}\left(\Theta_{j}\right)\right], A=\operatorname{Cov}\left(\beta\left(\Theta_{j}\right), \beta\left(\Theta_{j}\right)^{\prime}\right), b=E\left[\beta\left(\Theta_{j}\right)\right]$.

The credibility estimator for $\beta\left(\Theta_{j}\right)$ :

$\beta\left(\Theta_{j}\right)^{\text {Cred }}=Z_{j} \widehat{\beta}_{j}+\left(I-Z_{j}\right) b$, where $Z_{j}=A\left[A+s^{2}\left(X_{j}^{\prime} W_{j} X_{j}\right)^{-1}\right]^{-1}, \widehat{\beta}_{j}=\left(X_{j}^{\prime}\left(W_{j}\right)^{-1} X_{j}\right)^{-1} X_{j}^{\prime} W_{j} Y_{j}$.

\section{Parameter estimation:}

$$
\begin{aligned}
\widehat{b} & =\left(\sum_{j=1}^{J} Z_{j}\right)^{-1} \sum_{j=1}^{J} Z_{j} \widehat{\beta}_{j} \\
\widehat{s^{2}} & =\frac{1}{J} \sum_{j=1}^{J} \widehat{s_{j}^{2}} \text {, with } \widehat{s_{j}^{2}}=\frac{1}{n-K}\left(Y_{j}-X_{j} \widehat{\beta}_{j}\right)^{\prime}\left(Y_{j}-X_{j} \widehat{\beta}_{j}\right) ; \\
\widehat{A} & =\frac{1}{J-1} \sum_{j=1}^{J} Z_{j}\left(\widehat{\beta}_{j}-\widehat{b}\right)\left(\beta_{j}-\widehat{b}\right)^{\prime} .
\end{aligned}
$$

\section{Numeric Example}

Apply this method to Hachmeister's claims data.

State Indiv.coef.: $\widehat{\beta}_{j}=\left(X_{j}^{\prime}\left(W_{j}\right)^{-1} X_{j}\right)^{-1} X_{j}^{\prime} W_{j} Y_{j}$,

where $X=X_{j}=\left(\begin{array}{cc}1 & 1 \\ 1 & 2 \\ \vdots & \vdots \\ 1 & 12\end{array}\right), W_{j}=\left(\begin{array}{cccc}w_{j 1} & 0 & \cdots & 0 \\ 0 & w_{j 2} & \cdots & 0 \\ 0 & 0 & \cdots & w_{j, 12}\end{array}\right), Y_{j}=\left(\begin{array}{c}L_{j 1} \\ \vdots \\ L_{j, 12}\end{array}\right)$.

The results are shown in the table below:

\begin{tabular}{c|c|c|c|c|c}
\hline & state1 & state2 & state3 & state4 & state5 \\
\hline intercept & 1658.47243 & 1398.30252 & 1532.99872 & 1176.70407 & 1521.89933 \\
\hline slope coefficient & 62.39246 & 17.13975 & 43.30732 & 27.80702 & 11.87448 \\
\hline
\end{tabular}

TABLE 4: Regression coefficients Hachemeister's regression model

All the slope coefficients are positive, then there indeed exists a long-term increase in the data, which can also be seen from the regression lines (upward sloping) shown in figure 6 .

Estimating Insurance Premiums using Credibility 

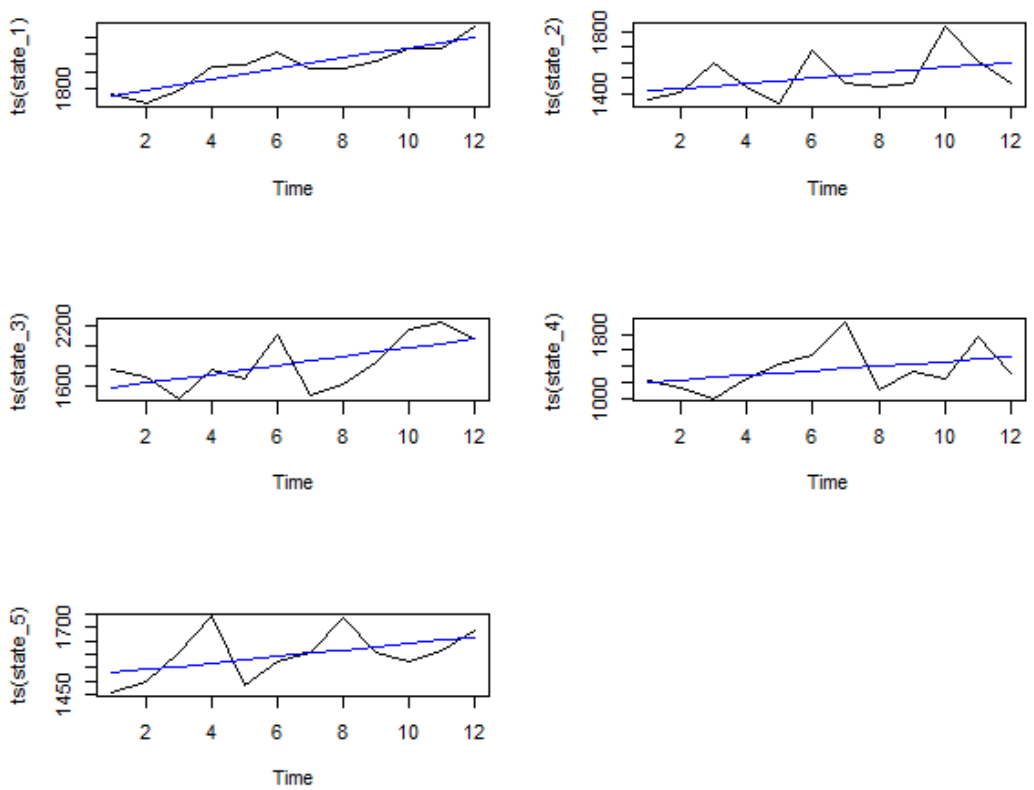

FiguRE 6: Weighted least square regressions

Within state variance: $\widehat{s^{2}}=\frac{1}{5} \sum_{j=1}^{5} \widehat{s_{j}^{2}}=49870187$,

where $\widehat{s_{1}^{2}}=121262869, \widehat{s_{2}^{2}}=30174010, \widehat{s_{3}^{2}}=52483869, \widehat{s_{4}^{2}}=24359005, \widehat{s_{5}^{2}}=21071182$;

State Adj.coef.:

\begin{tabular}{c|c|c|c|c|c}
\hline & state1 & state2 & state3 & state4 & state5 \\
\hline intercept & 1693.52313 & 1373.02958 & 1545.36429 & 1314.54855 & 1417.40928 \\
\hline slope coef. & 57.17147 & 21.34641 & 40.61014 & 14.80935 & 26.30721
\end{tabular}

TABLE 5: Regression coefficients Hachemeister's regression model

Finally, Premium $=$ Adj. intercept $+13 \times$ slope coef. :

\begin{tabular}{c|c|c|c|c|c}
\hline & state1 & state2 & state3 & state4 & state5 \\
\hline premium & 2436.75 & 1650.53 & 2073.30 & 1507.07 & 1759.40 \\
\hline
\end{tabular}

TABLE 6: Premiums Hachemeister's regression model

\subsection{Quantile Regression Credibility Model}

This section shows how quantiles could be incorporated into Hachemeister's model. Here only unweighted case will be considered. Assume that there are J contracts, for each contract $n$ years of claims experience, or other characteristics. Let $Q_{P}\left(y_{j t} \mid \Theta_{j}\right)(0<p<1)$ be the conditional quantile of $y_{j t}$ corresponding to the unobservable random risk parameter $\Theta_{j}$ and $Y_{j}=\left(y_{j 1}, \ldots, y_{j n}\right)^{\prime}$ an observable vector of risks.

$\mathbf{3 6} \mid \begin{aligned} & \text { Marble } \\ & \text { Research } \\ & \text { Papers }\end{aligned}$


Further assumptions:

(i) $\Theta_{1}, \Theta_{2}, \cdots$ are identically distributed, the pairs $\left(\Theta_{1}, Y_{1}\right), \cdots$ are independent;

(ii) Given $\Theta_{j}$,

$$
\underbrace{Q_{p}\left(Y_{j} \mid \Theta_{j}\right)}_{\mathrm{n} \times 1}=\underbrace{X_{j}}_{\mathrm{n} \times \mathrm{K}} \underbrace{\beta_{p}\left(\Theta_{j}\right)}_{\mathrm{K} \times 1}
$$

where

$\beta_{p}\left(\Theta_{j}\right)=$ vector of unknown coef ficients of length $K(K \leq n)$ for pth quantile, is considered as a variable, its distribution is determined by the structure of the collective;

$X_{j}=$ known fixed matrix of rank $K$;

$\operatorname{Cov}\left(\widehat{\beta_{p j}},{\widehat{\beta_{p j}}}^{\prime} \mid \Theta_{j}\right)=\sigma_{\xi_{p}}^{2}\left(\Theta_{j}\right)\left(X_{j}^{\prime} X_{j}\right)^{-1}$.

The structural parameters:

$s_{\xi_{p}}^{2}=E\left[\sigma_{\xi_{p}}^{2}\left(\Theta_{j}\right)\right], A_{p}=\operatorname{Cov}\left(\beta_{p}\left(\Theta_{j}\right), \beta_{p}\left(\Theta_{j}\right)^{\prime}\right), \beta_{p}=E\left[\beta_{p}\left(\Theta_{j}\right)\right]$.

The credibility estimator for $\beta\left(\Theta_{j}\right)$ :

$$
\beta_{p j}\left(\Theta_{j}\right)^{C r e d}=Z_{p j} \widehat{\beta_{p j}}+\left(I-Z_{p j}\right) \beta_{p}
$$

where

$Z_{p j}=A_{p}\left[A_{p}+s_{\xi_{p}}^{2}\left(X_{j}^{\prime} X_{j}\right)^{-1}\right]^{-1}, \quad \widehat{\beta_{p j}}=\left(X_{j}^{\prime} X_{j}\right)^{-1} X_{j}^{\prime} Y_{p j}$

$Z_{p j}$ is solution to the following minimization problem:

$$
\begin{aligned}
Q & =E\left(\left[\beta_{p}\left(\Theta_{j}\right)-{\widehat{B_{p j}}}^{C r e d}\right]^{\prime}\left[\beta_{p}\left(\Theta_{j}\right)-{\widehat{B_{p j}}}^{C r e d}\right]\right) \\
& =E\left(\left[\beta_{p}\left(\Theta_{j}\right)-\beta_{p}-Z_{p j}\left({\widehat{\beta_{p j}}}^{-}-\beta_{p}\right)\right]^{\prime}\left[\beta_{p}\left(\Theta_{j}\right)-\beta_{p}-Z_{p j}\left(\widehat{\beta_{p j}}-\beta_{p}\right)\right]\right)
\end{aligned}
$$

Let $\beta_{p}^{0}\left(\Theta_{j}\right)=\beta_{p}\left(\Theta_{j}\right)-\beta_{p}, \beta_{p j}^{0}=\widehat{\beta}_{p j}-\beta_{p}$, then the above equation becomes:

$$
\begin{aligned}
Q & =E\left(\left[\beta_{p}^{0}\left(\Theta_{j}\right)-Z_{p j} \beta_{p j}^{0}\right]^{\prime}\left[\beta_{p}^{0}\left(\Theta_{j}\right)-Z_{p j} \beta_{p j}^{0}\right]\right) \\
& =E\left(\beta_{p}^{0}\left(\Theta_{j}\right)^{\prime} \beta_{p}^{0}\left(\Theta_{j}\right)+\left(\beta_{p j}^{0}\right)^{\prime}\left(Z_{p j}\right)^{\prime} Z_{p j} \beta_{p j}^{0}-\left(\beta_{p}^{0}\left(\Theta_{j}\right)\right)^{\prime} Z_{p j} \beta_{p j}^{0}-\left(Z_{p j} \beta_{p j}^{0}\right)^{\prime} \beta_{p}^{0}\left(\Theta_{j}\right)\right)
\end{aligned}
$$

Using the product rule and differentiating with respect to the matrix $Z_{p j}$,

$$
\frac{d Q}{d Z_{p j}}=-2 E\left[\beta_{p}^{0}\left(\Theta_{j}\right)\left(\beta_{p j}^{0}\right)^{\prime}-Z_{p j} \beta_{p j}^{0}\left(\beta_{p j}^{0}\right)^{\prime}\right] \stackrel{\text { set }}{=} 0
$$

Estimating Insurance Premiums using Credibility Theory and Quantiles 
as

$$
\begin{aligned}
& E\left[\beta_{p}^{0}\left(\Theta_{j}\right)\left(\beta_{p j}^{0}\right)^{\prime}-Z_{p j} \beta_{p j}^{0}\left(\beta_{p j}^{0}\right)^{\prime}\right] \\
= & E\left(\left[\beta_{p}\left(\Theta_{j}\right)-\beta_{p}\right]\left[\beta_{p}\left(\Theta_{j}\right)-\beta_{p}\right]^{\prime}-Z_{p j}\left[\widehat{\beta}_{p j}-\beta_{p}\right]\left[\widehat{\beta}_{p j}-\beta_{p}\right]^{\prime}\right) \\
= & E\left(\left[\beta_{p}\left(\Theta_{j}\right)-\beta_{p}\right]\left[\beta_{p}\left(\Theta_{j}\right)-\beta_{p}\right]^{\prime}\right)-E\left(Z_{p j}\left[\widehat{\beta}_{p j}-\beta_{p}\right]\left[\widehat{\beta}_{p j}-\beta_{p}\right]^{\prime}\right) \\
= & \operatorname{Cov}\left(\beta_{p}\left(\Theta_{j}\right)\right)-Z_{p j} \operatorname{Cov}\left(\widehat{\beta}_{p j}\right) \\
= & \operatorname{Cov}\left(\beta_{p}\left(\Theta_{j}\right)\right)-Z_{p j}\left(E\left(\operatorname{Cov}\left[\widehat{\beta}_{p j} \mid \Theta_{j}\right]\right)+\operatorname{Cov}\left(E\left[\widehat{\beta}_{p j} \mid \Theta_{j}\right]\right)\right)
\end{aligned}
$$

then $Z_{p j}=\operatorname{Cov}\left(\beta_{p}\left(\Theta_{j}\right)\right)\left(E\left(\operatorname{Cov}\left[\widehat{\beta}_{p j} \mid \Theta_{j}\right]\right)+\operatorname{cov}\left(E\left[\widehat{\beta}_{p j} \mid \Theta_{j}\right]\right)\right)^{-1}$

\section{Parameter estimation:}

$$
\begin{aligned}
& \widehat{\beta_{p j}}=\underset{\beta_{p}}{\operatorname{argmin}}\left(\sum_{i: y_{i} \geq b} p\left|y_{j i}-x_{j i}^{\prime} \beta_{j}\right|+\sum_{i: y_{j}<b}(1-p)\left|y_{j i}-x_{j i}^{\prime} \beta_{j}\right|\right) ; \\
& \widehat{\beta_{p}}=\frac{1}{J} \sum_{j=1}^{J} \widehat{\beta_{p j}} ; \\
& \widehat{A_{p}}=\frac{1}{J-1} \sum_{j=1}^{J}\left(\widehat{\beta}_{p j}-\widehat{\beta_{p}}\right)\left(\widehat{\beta}_{p j}-\widehat{\beta_{p}}\right)^{\prime}-\frac{1}{J} \sum_{j=1}^{J} \widehat{\sigma_{\xi_{p}}^{2}}\left(\Theta_{j}\right)\left(X_{j}^{\prime} X_{j}\right)^{-1} ; \\
& \operatorname{Cov}\left(\widehat{\beta_{p j}} \mid \Theta_{j}\right)=\sigma_{\xi_{p}}^{2}\left(\Theta_{j}\right)\left(X_{j}^{\prime} X_{j}\right)^{-1}=\frac{p(1-p)}{n\left(f_{\xi_{p}}\right)^{2}}\left(X_{j}^{\prime} X_{j}\right)^{-1} .
\end{aligned}
$$

$\sigma_{\xi_{p}}^{2}\left(\Theta_{j}\right)$ is estimated using order statistic estimation:

$$
\widehat{\sigma_{\xi_{p}}^{2}}=\frac{1}{J} \sum_{j=1}^{J} \frac{\widehat{\omega_{p}\left(\Theta_{j}\right)}}{n}
$$

where

$$
\widehat{\omega_{p}\left(\Theta_{j}\right)}=\frac{n^{2}\left(y_{\lfloor n p+l\rfloor}-y_{\lfloor n p-l\rfloor}\right)^{2}}{4 Z_{1-\alpha / 2}^{2}}, l=Z_{1-\alpha / 2} \sqrt{n p(1-p)} .
$$

\section{Numeric Example}

Apply this method to Hachmeister's claims data.

Premiums estimated:

\begin{tabular}{l|c|c|c|c|c}
\hline & state1 & state2 & state3 & state4 & state5 \\
\hline premium & 2098.915 & 1684.715 & 1960.715 & 1618.415 & 1762.39 \\
\hline
\end{tabular}

TABLE 7: Premiums Quantile regression credibility model 


\section{Effect of outliers on the models}

The premiums estimated in previous sections are summarized in Table 8. It is noticeable from the summary statistics in the table that for each state premium estimated using Hachemeister's regression model is greater than using Bühlman-Straub model, which is due to the fact that Hachemeister's regression model considers long-term increas in next period, while Bühlman-Straub model doesnot.

\begin{tabular}{l|ccccc}
\hline & premium1 & premium2 & premium3 & premium4 & premium5 \\
\hline Bühlman-Straub & 2055.17 & 1523.71 & 1793.44 & 1442.97 & 1603.29 \\
\hline Hachemeister regression & 2436.75 & 1650.53 & 2073.30 & 1507.07 & 1759.40 \\
\hline Quantile(0.5) credi. & 1521.66 & 1681.40 & 1602.87 & 1730.95 & 1644.13 \\
\hline Quantile regression credi.(0.5) & 2098.92 & 1684.72 & 1960.72 & 1618.42 & 1762.39 \\
\hline
\end{tabular}

TABlE 8: Summary premiums.

In order to see the effect of outliers on these four models, Hachemeister's claims data is revised in two steps. The results are presented in Table 9.

(A) Increase the last observation of state1 to 100000

\begin{tabular}{c|c|c|c|c|c} 
& premium1 & premium2 & premium3 & premium4 & premium5 \\
\hline Bühlman-Straub & 10895.74 & 1511.25 & 1805.87 & 1353.10 & 1599.84 \\
\hline Hachemeister regression & - & - & - & - & - \\
\hline
\end{tabular}

(B) Increase the last observation of state1, state2 and state3 to 100000

\begin{tabular}{c|c|c|c|c|c} 
& premium1 & premium2 & premium3 & premium4 & premium5 \\
\hline Bühlman-Straub & 10895.75 & 10727.69 & 9799.37 & 1353.31 & 1599.87 \\
\hline Hachemeister regression & - & - & - & - & - \\
\hline Quantile(0.5) & 1477.74 & 1698.73 & 1590.08 & 1767.28 & 1647.18 \\
\hline
\end{tabular}

TABLE 9: Summary premiums for revised data.

First, increase the last observation of state 1 to 100000 . The results indicate that the most significant change is in the premium estimated for state1 using the BühlmannStraub model. To point out, $\tilde{a}$ in the Bühlmann-Straub model is negative in this case, so set $\tilde{a}=(J-1) \times \tilde{s}^{2}$. Furthermore, the Hachemeister's regression model is invalid under this circumstance, because the design matrix is not invertible, hence it cannot be used to develop a regression model. Note that the premiums estimated using Quantile credibility model and Quantile regression credibility model are omitted in the table, as there is no changes to these premiums.

Next, also increase the last observation of state 2 and state 3 to 100000 . Similarly, the premiums estimated for state 1 , state 2 and state 3 are largely affected using the Bühlmann-Straub model. Moreover, $\tilde{a}$ in the Bühlmann-Straub model is negative and the Hachemeister's regression model remains invalid. What is particlarly interersting is

Estimating Insurance Premiums using Credibility 
that there is just a small increase to premiums estimated using Quantile credibility model, but no changes to the premiums estimated using Quantile regression credibility model. It can be clear from the results that outliers have less impact on Quantile credibility model and Quantile credibility regression model than on the Bühlmann-Straub model and the Hachemeister's regression model.

\section{Conclusion}

Despite the fact that the models are applied to Hachemeister's data set which is different from Pitselis' paper, similar conclusions can be drawn. They are summarized as follows. Firstly, incorporating with quantiles enables to estimate premiums as well as changes in these premiums at different points of the claims distributions. Secondly, Quantile credibility model and Quantile credibility regression model are less sensitive to outlying data. Finally, as the claims distributions are in general heavy-tailed and skewed to the right, quantile estimation is more desirable than least squares estimation in the context of the insurance industry. However, it is important to note that there is a shortcoming in the Hachemeister's data set. That is, the size of Hachemeister's data set is relatively small, which could influence the impact of the outliers on the models. Therefore, further researches such as applying these models to data with a larger sample size are recommended.

\section{References}

[1] Bühlmann, H., Gisler, A. (2005). A course in Credibility Theory and its Applications. Heidelberg: Springer.

[2] Kaas, R. and et al (2001). Modern Actuarial Risk Theory. Dordrecht: Kluwer Academic publishers.

[3] Koenker, R. and Bassett, G. (1978). Regression Quantiles. Econometrica, 46, 33-50.

[4] Greene, W.H. (2012). Econometric Analysis. 7th ed. Harlow: Pearson Education Limited.

[5] Pitselis, G. (2013). Quantile credibility models. Insurance: Mathematics and Economics, 52, 477-489.

\footnotetext{
40 \begin{tabular}{l|l} 
Marble \\
Research \\
Papers
\end{tabular}
} 